\title{
Alta correlación entre el consumo de ciprofloxacina y la prevalencia de Klebsiella pneumoniae productora de $\beta$-lactamasas de espectro extendido
}

\author{
Joaquín Bermejo, Blanca Bencomo, Nora Arnesi, Patricia Lesnaberes, Noemí Borda y Rodolfo Notario
}

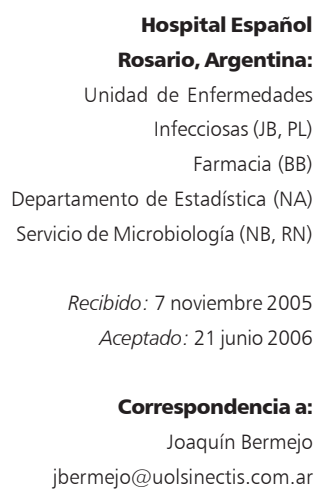

Hospital Español Rosario, Argentina: nfecciosas (JB, PL) Farmacia (BB) Departamento de Estadística (NA) Servicio de Microbiología (NB, RN)

Recibido: 7 noviembre 2005 Correspondencia a: bermejo@uolsinectis.com.ar

\section{High relationship between ciprofloxacin use and prevalence of ESBL Klebsiella pneumoniae}

Background: To assess the relationship between ciprofloxacin use and the prevalence of extended spectrum betalactamases (ESBL) Klebsiella pneumoniae. Patients and Methods: Semestral mean values regarding use of antibiotic and prevalence of ESBL Kp were compared during 9 semesters using linear regression and coefficient of correlation. Results: The only statistically significant correlation was ciprofloxacin use and $\operatorname{ESBL}(+) K$. pneumoniae prevalence, with a coefficient of correlation of 0.86 and $p=0.0027$ using linear regression. Conclusions: Ciprofloxacin use must be taking into account when considering infection control programs due to high prevalence rates of $\operatorname{ESBL}(+) K$. pneumoniae in the hospital setting.

Key words: Klebsiella pneumoniae; extended spectrum betalactamases; ciprofloxacin.

Palabras claves: Klebsiella pneumoniae; $\beta$-lactamasas de espectro extendido; ciprofloxacina.

\section{Introducción}

$K$ lebsiella pneumoniae es uno de los patógenos nosocomiales prevalentes. Desde hace más de 20 años se reconoce en esta bacteria la capacidad de resistir la acción de cefalosporinas de espectro extendido y aztreonam, gracias a la producción de enzimas conocidas como $\beta$-lactamasas de espectro extendido (BLEE) $)^{1,2}$. Numerosos autores han evaluado los factores relacionados con la aparición y diseminación de estas $\operatorname{cepas}^{3-10}$. Independientemente de los diseños de esos estudios, los escenarios y la situación epidemiológica, la inmensa mayoría acuerda en considerar que el uso previo de antimicrobianos es un factor de riesgo para la adquisición nosocomial de $K$. pneumoniae BLEE $(+)$. Es justamente el uso de cefalosporinas de amplio espectro, tales como ceftazidima, cefotaxima o ceftriaxona, repetidamente reconocido como factor asociado, por encima de otros antimicrobianos pertenecientes a otras familias químicas. A la luz de los actuales conocimientos, la fuerza de esa asociación parece tan probada que los hospitales con diseminación epidémica de $K$. pneumoniae BLEE $(+)$ deberían adoptar, entre otras, medidas de restricción del uso de cefalosporinas de amplio espectro para controlar la situación. Desafortunadamente la restricción de cefalosporinas de amplio espectro en los hospitales, sobre todo en las áreas críticas, lleva al uso de otros antibacterianos, de generación más reciente, muchas veces más costosos que impactan desfavorablemente en la ecología del medio, promoviendo la aparición de más resistencia bacteriana ${ }^{11,12}$ o de otras bacterias multirresistentes ${ }^{13,14}$. Algunos autores ${ }^{15} \mathrm{y}$ nosotros mismos ${ }^{10}$ hemos hallado que el uso previo de ciprofloxacina, y no el de cefalosporinas de amplio espectro, puede comportarse como un factor de riesgo asociado a la adquisición de infecciones debidas a $K$. pneumoniae BLEE $(+)$; por lo que parece prudente que antes de emprender una política de restricción los hospitales analicen la posible relación de la prevalencia de $K$. pneumoniae BLEE $(+)$ con el consumo de otros compuestos antibacterianos. El objetivo de este estudio fue explorar la relación que, en el transcurso de 4 años y medio, mantuvieron el uso de ciprofloxacina y de cefalosporinas de tercera generación con la evolución en la prevalencia de resistencia mediada por BLEE en cepas de $K$. pneumoniae provenientes de aislados clínicos de pacientes ingresados a nuestro hospital.

\section{Material y Métodos}

Este es un estudio observacional, analítico y transversal, realizado en un hospital general de pacientes agudos, con 200 camas, de complejidad intermedia, que tiene 9.500 ingresos anuales promedio, de los 
cuales 53\% son quirúrgicos. El hospital no cuenta con programas de trasplante de órganos y no tiene unidad de quemados. Los datos de consumo de antimicrobianos fueron suministrados desde la base de datos de farmacia. Las únicas cefalosporinas de amplio espectro disponibles en la farmacia de nuestro hospital son cefotaxima, ceftriaxona y ceftazidima. Las unidades de consumo están expresadas en dosis definida diaria (DDD) por 1.000 pacientes-día. La DDD para ciprofloxacina fue $0,8 \mathrm{gr}$; ceftazidima 6 gr; cefotaxima 4 gr; ceftriaxona 1 gr. El servicio de microbiología integra la red WHONET. Las pruebas de identificación, así como la determinación de susceptibilidad, se hicieron según métodos estandarizados ${ }^{16,17}$. La producción de $\beta$-lactamasas de espectro extendido se determinó por el test de sinergia del doble disco ${ }^{18}$. Las tasas de resistencia bacteriana fueron obtenidas desde la base de datos de microbiología (WHONET 5.3, OMS) a través de una función propia del software que calcula el porcentaje de cepas resistentes (BLEE+) sobre el total de cepas de $K$. pneumoniae aisladas en muestras clínicas representativas durante el período de estudio. Utilizamos el coeficiente de correlación de Pearson $(r)$, una medida de asociación lineal entre dos variables cuantitativas y regresión lineal, para explorar el grado de asociación entre las variables (consumo de antimicrobianos y resistencia bacteriana) a lo largo del período de estudio. Se calculó el coeficiente de Pearson al cuadrado $\left(r^{2}\right)$ para estimar qué porcentaje de la variabilidad de la resistencia mediada por BLEE (variable dependiente) se explica si se conocen los valores de consumo de antimicrobianos (variable independiente). Se tomaron los consumos promedios semestrales para evitar las evoluciones erráticas observadas en las tasas de consumo que se observan al analizar períodos más cortos de tiempo. Para cada semestre se obtuvo la correspondiente tasa de resistencia, mediada por BLEE entre los aislados de $K$. pneumoniae. Para el análisis de los datos se utilizó el programa SAS-PC (versión 6.12, SAS Institute, Cary, NC).

\section{Resultados}

Se analizaron 9 semestres (julio 1999-diciembre 2003) en los que se aislaron 234 cepas de $K$. pneumoniae en 205 pacientes hospitalizados. Los aislados provenían de muestras de orina $(58,5 \%)$, heridas quirúrgicas $(16,2 \%)$, sangre $(12,4 \%)$ y otras $(12,9 \%)$. El $21,8 \%$ de las muestras fue extraído a pacientes que estaban internados en unidades de cuidados críticos, mientras que el resto estaba alojado en unidades de internación general. La estadía promedio de cada paciente, para el período de tiempo del estudio, fue de 3,8 días.
La tasa de incidencia de infecciones debidas a $K$. pneumoniae BLEE (+) para el período fue de 1,06 episodios/1.000 pacientes día. Un $67 \%$ de las $K$. pneumoniae fueron productoras de BLEE y $44 \%$ resistentes a ciprofloxacina. El valor de $r$ fue de 0,86 al explorar el consumo de ciprofloxacina con las tasas de prevalencia de K. pneumoniae BLEE (+) (Figura 1), mientras que en el análisis de regresión lineal se obtuvo un valor de $\mathrm{p}=0,0027$ sobre un nivel de confianza de $99 \%$ (Tabla 1), siendo el $r^{2}$ de $74,5 \%$. En el gráfico de dispersión de puntos se observa una distribución lineal de las variables (Figura 2). Los valores de $r$ y los resultados de la regresión lineal, para el consumo total de cefalosporinas de tercera generación (Figura 3), así como para ceftazidima, cefotaxima y ceftriaxona (Tabla 1), no mostraron significación.

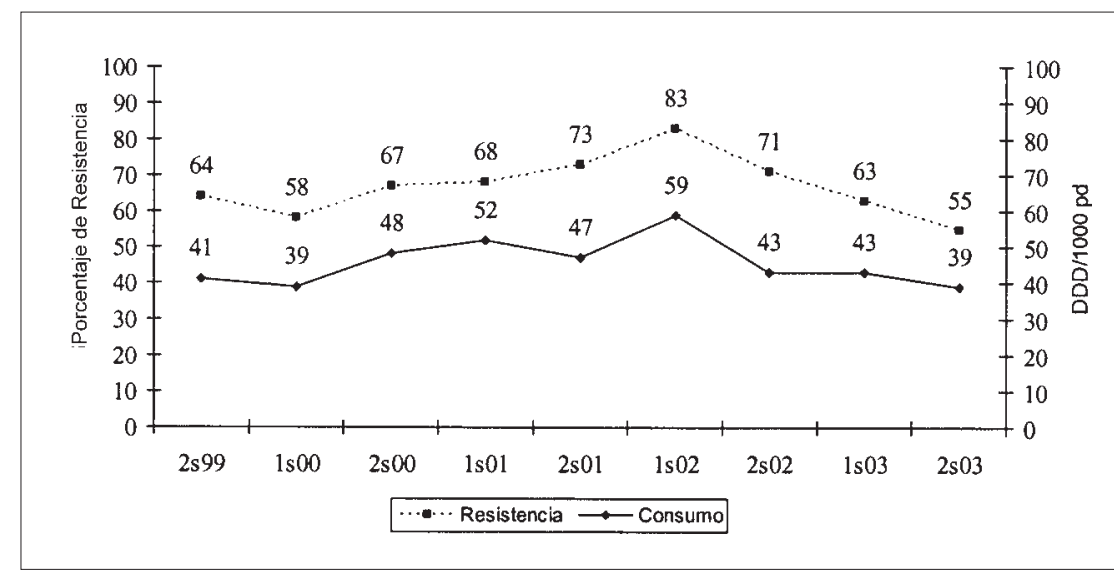

Figura 1. Evolución de la prevalencia de Klebsiella penumoniae BLEE (+) y su correlación con el consumo de ciprofloxacina.

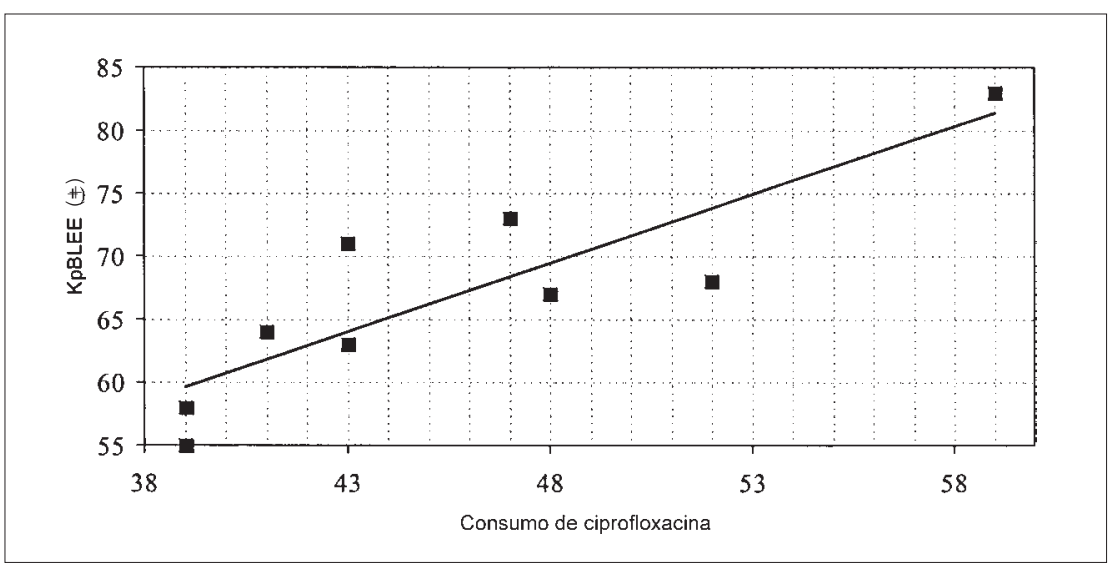

Figura 2. Dispersión de puntos entre el consumo de ciprofloxacina y resistencia de Klebsiella penumoniae mediadas por BLEE. 


\begin{tabular}{|c|c|c|}
\hline Fármaco & Valor $r$ & Valor $p$ \\
\hline Ciprofloxacina & 0,86 & 0,0027 \\
\hline Cefalosporinas $3^{\text {a }}$ gen & 0,19 & 0,61 \\
\hline Ceftazidima & $-0,25$ & 0,49 \\
\hline Cefotaxima & 0,17 & 0,65 \\
\hline Ceftriaxona & 0,27 & 0,47 \\
\hline \multicolumn{3}{|c|}{$\begin{array}{l}\text { Cefalosporinas } 3^{a} \text { gen: Total de las cefalosporinas de tercera } \\
\text { generación. } \\
\text { Valor } r \text { : Coeficiente de correlación de Pearson. } \\
\text { Valor p: Significación de la regresión lineal }\end{array}$} \\
\hline
\end{tabular}

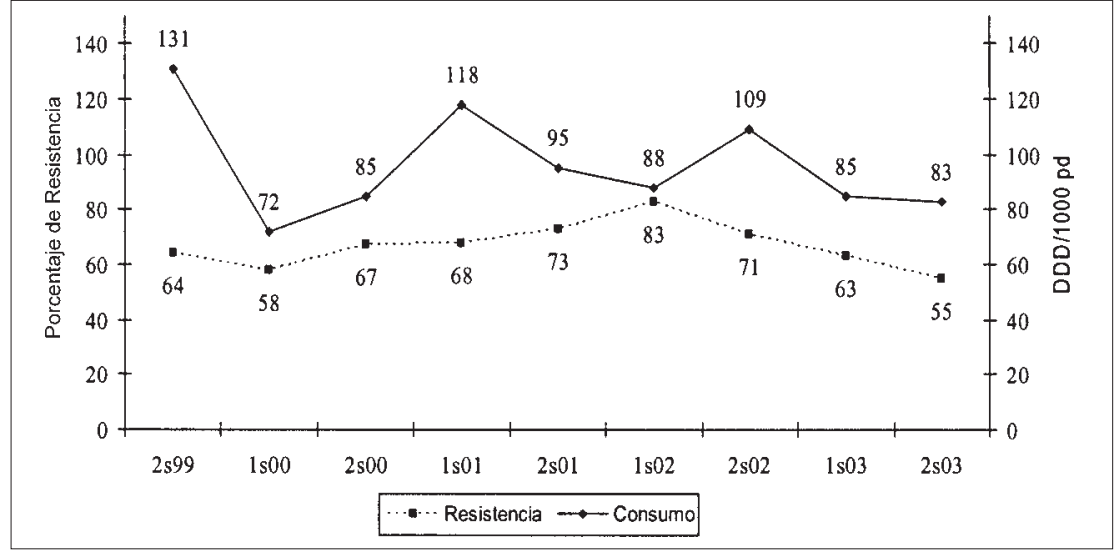

Figura 3. Evolución de la prevalencia de Klebsiella penumoniae BLEE $(+)$ y su correlación con el consumo de cefalosporinas de $3^{\text {a }}$ generación.

\section{Discusión}

El uso de antimicrobianos ha sido reconocido como uno de los factores responsables de los cambios temporales observados en la susceptibilidad bacteriana dentro de los hospitales. Reducir la presión selectiva es uno de los puntos ineludibles en las estrategias para controlar la presencia nosocomial de bacterias resistentes. Estas políticas de antimicrobianos tienen por objetivo optimizar su uso. El ideal es consumir sólo lo imprescindible, utilizando antimicrobianos de reducido espectro de acción y de bajo costo. Sin embargo, múltiples razones hacen difícil alcanzar ese objetivo.

La alta prevalencia de un patógeno nosocomial resistente a un determinado fármaco podría motivar una respuesta refleja: restringir el uso de dicho fármaco. En ese sentido hay experiencias que muestran resultados dispares. Así, Peña demostró que la restricción de cefalosporinas de espectro extendido en las unidades de cuidados críticos de un hospital universitario de Barcelona $^{19}$, permitió controlar un brote de $K$. pneumoniae BLEE (+), constituyéndose en un ejemplo a favor de tal política. Sin embargo, de modo opuesto, la restricción del uso de vancomicina, en la experiencia de Lautembach ${ }^{20}$, no se acompañó de una reducción en las tasas de Enterococcus resistentes a vancomicina, $\mathrm{y}$ en cambio, lo consiguieron tras restringir el consumo de clindamicina.

Numerosos estudios han explorado la relación entre el consumo de, y la resistencia a un determinado antimicrobiano. Sin embargo, la existencia simultánea de distintos mecanismos de resistencia en las llamadas bacterias multirresistentes, podría promover el fenómeno de co-selección, por el cual el uso de un antimicrobiano de alto consumo en el hospital selec- cionaría subpoblaciones de bacterias resistentes, también, a otros fármacos de estructura química diferente. Así, se conoce que cepas de Pseudomonas aeruginosa resistentes a carbapenems fueron ocasionalmente seleccionadas por fluoroquinolonas ${ }^{21}$. Esas cepas de $P$. aeruginosa tenían mutaciones en el gen $n f x c$ (mexT) con expulsión activa y una simultánea disminución de la permeabilidad que confería resistencia a fluoroquinolonas y carbapenemes.

El análisis de las razones por las cuales hallamos correlación entre las tasas de resistencia en $K$. pneumoniae mediada por BLEE y el consumo de ciprofloxacina y no con el consumo de cefalosporinas de tercera generación, nos hubiese exigido realizar estudios de biología molecular para definir clonalidad de las cepas aisladas y profundizar en sus mecanismos de resistencia a los antimicrobianos no $\beta$-lactámicos, pero estos recursos no están disponibles en nuestro medio. Aunque mutaciones cromosómicas son las principales responsables de la resistencia a quinolonas en Enterobacteriaceae, éstas pueden también ocurrir, entre otros, a través de determinantes genéticos (Qnr) codificados por plásmidos ${ }^{22}$. En la literatura científica ${ }^{23}$ está descrita la presencia de cepas bacterianas que expresan BLEE de diferentes tipos (SHV-5, -7, -12, CTX-M14, -15 y VEB-1) concomitantemente a Qnr. Esta asociación de genes de resistencia a antimicrobianos podría explicar el fenómeno de resistencia asociada entre fluroroquinolonas y BLEE en Enterobacteriaceae y por tanto, la capacidad de co-selección entre ambos tipos de resistencia y fármacos. Nuestra hipótesis es que quizás en nuestro hospital han circulado cepas con tales características que conjuntamente con un uso elevado de ciprofloxacina han proporcionado el escenario adecuado para tal hallazgo. 
Algunos autores ${ }^{25}$ han encontrado divergencia entre los resultados de dos tipos de estudios epidemiológicos que intentan relacionar el uso de antimicrobianos y la resistencia bacteriana; los casos y controles, por un lado y los estudios que correlacionan la densidad de consumo con las tasas de resistencia, por el otro. En nuestra experiencia, la alta correlación entre la evolución del consumo de ciprofloxacina y la prevalencia de K. pneumoniae BLEE (+) es un hallazgo significativo que coincide con una observación previa individual. En un estudio de casos y controles $^{10}$ habíamos concluido que el uso previo de ciprofloxacina, y no el de cefalosporinas de amplio espectro, se comportaba como un factor de riesgo independiente para que los pacientes que adquirían una infección debida a $K$. pneumonaie, lo hicieran por una cepa BLEE $(+)$. En ese estudio, $78 \%$ de las $K$. pneumoniae BLEE $(+)$ eran resistentes a ciprofloxacina, mientras que sólo lo eran $18 \%$ de las $K$. pneumoniae no productoras de BLEE.

El valor $r(0,86)$, entre el consumo de ciprofloxacina y la prevalencia de $K$. pneumoniae BLEE $(+)$ muestra una correlación positiva elevada (cercana a +1 ), no observada con los otros antimicrobianos explorados, indicando que están relacionados directamente, esto es, cuando el consumo de ciprofloxacina tiende a incrementarse también lo hace la prevalencia de $K$. pneumoniae BLEE $(+)$.

Finalmente, es necesario explorar la correlación entre el consumo de antimicrobianos y la resistencia bacteriana antes de que un hospital emprenda un pro- grama de restricción de antimicrobianos, focalizado o global, a los fines de controlar la problemática de la multirresistencia. Esa exploración no debe limitarse a estudiar la relación de consumo y resistencia a un determinado fármaco ${ }^{25}$, sino debe extenderse a binomios (antimicrobiano/resistencia) no relacionados entre sí, en función de detectar fenómenos de co-selección. Es importante tener en cuenta que la alta correlación hallada entre las dos variables no implica una relación causal.

\section{Resumen}

Fundamento: Evaluar la correlación entre el consumo de cefalosporinas de tercera generación y ciprofloxacina con la prevalencia de cepas de Klebsiella pneumoniae productoras de $\beta$-lactamasas de espectro extendido (BLEE). Pacientes y Métodos: Los valores promedios semestrales, correspondientes a consumo y prevalencia se compararon durante 9 semestres, usando coeficiente de correlación y regresión lineal. Resultados: La única asociación que resultó estadísticamente significativa, fue la correspondiente al consumo de ciprofloxacina y $K$. pneumoniae BLEE (+), con un coeficiente de correlación de 0,86 y una $p$ de 0,0027, en el análisis de regresión lineal. Conclusiones: El consumo de ciprofloxacina debe ser tenido en cuenta al momento de establecer programas de control de infecciones frente a elevadas tasas de prevalencia de $K$. pneumoniae productoras de BLEE en un hospital.

\section{Referencias}

1.- Sirot D, Sirot J, Labia R, Moraud A, Courvalin P, Darfeuille-Michaud A, et al. Transferable resistance to third generation cephalosporins in clinical isolates of Klebsiella pneumoniae: identification of CTX-1, a novel beta-lactamase. J Antimicrob Chemother 1987; 20: 323-34.

2.- Brun-Buisson C, Legrand P, Philippon A, Montravers F, Ansquer M, Dural J.

Transferable enzymatic resistance to thirdgeneration cephalosporins during nosocomial outbreak of multiresistant Klebsiella pneumoniae. Lancet 1987; 2: 302-6.

3.- Harris AD, Karchmer T B, Carmeli Y, Samore M. Methodological principles of case-control studies that analyzed risk factors for antibiotic resistance: A systematic review. Clin Infect Dis 2001; 32: 1055-61.
4.- Schiappa D A, Hayden M K, Matushek M G, Hashemi F N, Sullivan J, Smith K Y, et al. Ceftazidime-resistant Klebsiella pneumoniae and Escherichia coli bloodstream infection: A case-control and molecular epidemiologic investigation. J Infect Dis 1996; 17: 529-36.

5.- Peña $C$, Pujol M, Ricart A, Ardanuy $C$, Ayats J, Liñares J, et al. Risk factors for faecal carriage of Klebsiella pneumoniae producing extended spectrum beta-lactamase (ESBL-Kp) in the intensive care unit. J Hosp Infect 1997; 35: 9-16.

6.- Asensio A, Oliver A, González-Diego P, Baquero F, Pérez-Díaz JC, Ros $\mathrm{P}$, et al. Outbreak of a multiresistant Klebsiella pneumoniae strain in an intensive care unit: antibiotic use as risk factor for colonization and infection. Clin Infect Dis 2000; 30: $55-60$

7.- Arpin C, Rogues A M, Kabouche S,
Boulard G, Quesnel C, Gachie JP, et al. Prospective survey of colonization and infection caused by SHV-4 producing Klebsiella pneumoniae in a neurosurgical intensive care unit. Epidemiol Infect 2000; 124: 401-8

8.- Wiener J, Quinn J P, Bradford P A, Goering RV, Nathan C, Bush K, et al Multiple antibiotic-resistant Klebsiella and Escherichia coli in nursing homes. JAMA 1999; 281: 517-23.

9.- Lautenbach E, Patel J B, Bilker W B, Edelstein P H, Fishman NO. Extendedspectrum $\beta$-lactamase-producing Escherichia coli and Klebsiella pneumoniae: Risk factors for infection and impact of resistance on outcomes. Clin Infect Dis 2001; 32: 1162-71.

10.- Bermejo J, Lesnaberes P, Arnesi N, Gianello M, Notario R, Borda N, et al. Factores asociados a infecciones por Klebsiella 
pneumoniae resistentes a ceftacidima. Enferm Infecc Microbiol Clin 2003; 21: 72-6.

11.- McGowan J E. Antimicrobial resistance in hospital organisms and its relation to antibiotic use. Rev Infect Dis 1983; 5: 1033-48

12.- Oewns R C, Rice L. Hospital-based strategies for combating resistance. Clin Infect Dis 2006; 42: S173-81.

13.- Sanyal S C, Mokaddas E M. The increase in carbapenem use and emergence of Stenotrophomonas maltophilia as an important nosocomial pathogen. J Chemother 1999; 11: 28-33.

14.- Landman D, Chockalingam M, Quale J M. Reduction in the incidence of methicillinresistant Staphylococcus aureus and ceftazidime-resistant Klebsiella pneumoniae following changes in a hospital antibiotic formulary. Clin Infect Dis 1999; 28: 1062-6.

15.- Paterson D, Mulazimoglu L, Casellas J M, Ko W, Goossenns H, Gottberg A V, et al. Epidemiology of ciprofloxacin resístance and its relationship to extended spectrum beta lactamase production in $K$ pneumoniae isolates causing bacteremia. Clin Infect Dis 2000;
30: 473-8.

16. - Farmer III J J. Chapter 41:

Enterobacteriaceae: Introduction and identification. In Murray, P.R. (Editor in chief), Baron EJ, Jorgensen JH, Pfaller M A, Yolken RH. (ed). Manual of Clinical Microbiology 8th ed. ASM Press, Washington 2003; 636-53.

17. - CLSI Clinical and Laboratory Standards Institute. Performance Standards for Antimicrobial Susceptibility Testing. M100S15. Vol $25 \mathrm{~N}^{\mathrm{o}} 1$. Wayne. 2005.

18.- Legrand P, Fournier G, Bure A, Jarlier V, Nicolás $\mathrm{M} \mathrm{H}$, Decre $\mathrm{D}$, et al. Detection of extended broad-spectrum beta-lactamases in Enterobacteriaceae in four french hospitals. Eur J Clin Microbiol Infect Dis 1989; 8: 527-9.

19.- Peña C, Pujol M, Ardanuy C, Ricart A, Pallares R, Liñares J, et al. Epidemiology and successful control of a large outbreak due to Klebsiella pneumoniae producing extended spectrum beta-lactamases. Antimicrob Agents Chemother 1998; 42: 53-8.

20.- Lautenbach E, LaRosa L A, Marr A M, Nachamkin I, Bilker W B, Fishman N O. Changes in the prevalence of vancomycinresistant Enterococci in response to antimicrobial formulary interventions: Impact of progressive restrictions on use of vancomycin and third-generation cephalosporins. Clin Infect Dis 2003; 36: 440-6.

21.- Ochs M M, Mc Cusker M P, Bains M, Hancock R E. Negative regulation of the Pseudomonas aeruginosa outer membrane porin OprD selective for imipenem and basic amino acids. Antimicrob Agents Chemother 1999; 43: 1085-90

22.- Martínez-Martínez L, Pascual A, Jacoby GA. Quinolone resistance from a transferable plasmid. Lancet 1998; 351: 797-9.

23.- Corkill J E, Anson J J, Hart C A. High prevalence of the plasmid-mediated quinolone resistance determinant qnrA in multidrug-resistant Enterobacteriaceae from blood cultures in Liverpool, UK. J Antimicrob Chemother 2005; 56: 1115-7.

24. - Harbarth S, Harris A D, Carmeli Y, Samore M. Parallel analysis of individual and aggregated data on antibiotic exposure and resistance in Gram-negative bacilli. Clin Infect Dis 2001; 33: 1462-8.

25.- Friedrich L V, White R L, Bosso J A. Impact of use of multiple antimicrobial on changes in susceptibility of Gram-negative aerobes. Clin Infect Dis 1999; 28: 1017-24. 